\title{
Evidence of the spin Seebeck effect in Ni-Zn ferrites polycrystalline slabs
}

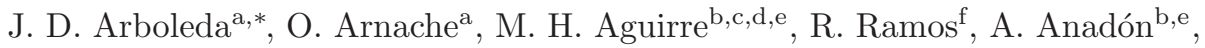 \\ M. R. Ibarra ${ }^{\mathrm{b}, \mathrm{c}, \mathrm{d}, \mathrm{e}}$ \\ ${ }^{a}$ Instituto de Física, Universidad de Antioquia, A.A. 1226, Medellín-Colombia \\ ${ }^{b}$ Instituto de Nanociencia de Aragón, Universidad de Zaragoza, E-50018 Zaragoza-Spain \\ ${ }^{c}$ Laboratorio de microscopías avanzadas, Universidad de Zaragoza, Zaragoza, Spain \\ ${ }^{d}$ Fundación INA, E-50018 Zaragoza-Spain \\ ${ }^{e}$ Departamento de Física de la Materia Condensada, Universidad de Zaragoza, E-50009 \\ Zaragoza-Spain \\ ${ }^{f}$ WPI Advanced Institute for Materials Research, Tohoku University, Sendai-Japan
}

\begin{abstract}
We report on the observation of the spin Seebeck effect in Ni-Zn ferrites slabs with different Zn concentration. All samples have a spinel structure confirmed by XRD and TEM. We fully characterize the magnetic properties by VSM and Mössbauer spectroscopy. Samples exhibit a nonmonotonic magnetization behavior depending on the structural inversion parameter, however we found a spin Seebeck response voltage of about $25.5 \mathrm{nV} / \mathrm{K}$ independent of the magnetization and the inversion degree.
\end{abstract}

Keywords: , Spin seebeck effect, Spin caloritronics, Ni-Zn ferrites

\section{Introduction}

The generation of spin current induced by an applied thermal gradient via the spin Seebeck effect (SSE)[1] is one of the most remarkable effects in the growing field of spin caloritronics[2, 3]. The SSE has been observed in a great va-

5 riety of materials with different electrical (conductors[4] and insulators[5, 6]) and magnetic (ferromagnetic or ferrimagnetic, weak ferromagnetic[7], antiferromagnetic[8,

\footnotetext{
* Corresponding author

Email address: juan.arboledaj@udea.edu.co (J. D. Arboleda)
} 
9], and paramagnetic[10]) properties, both in thin film, including heterostructures[11, 12], and bulk materials $[13,5,14,15,16]$.

The SSE is a thermoelectric effect mediated by the spin degree of freedom. When a magnetic material (FM) is subjected to a temperature gradient generates a spin current parallel to the thermal gradient. This spin current is detected in an attached normal metal (NM), with high spin-orbit coupling, as an electromotive force by means of the inverse spin Hall effect (ISHE)[17].

Despite the intense work being carried out by different research groups, the

15 mechanism underlying the SSE is still under discussion[18, 19, 20, 21, 22]. The role of the magnetic order, the different length scales of the materials involved, effects at the interface, the surface magnetization, among others, are still active research lines[23, 24, 25], e. g. in YIG, Aqeel et al.[26] demonstrated that surface properties strong influence the SSE response by preparing samples with different surface quality. On the other hand, Kalappattil et al.[27], very recently, reported surface magnetic anisotropy dependence in bulk YIG slabs, showing the importance of surface magnetization in the SSE measurements.

We recently reported the observation of SSE in the weak ferromagnetic Zn ferrite[7] with considerable voltage response despite the negligible magnetiza-

25 tion. This result suggest that the SSE signal is independent of the FM magnetization saturation. The SSE in these types of ferrites might be also influenced by the surface magnetization in the FM.

The SSE in the longitudinal configuration has been investigated in a variety of spinel ferrites such as Cobalt [28, 29], Nickel[30,31] and Zinc ferrites[7], espe-

so cially in thin films. In this paper we systematically investigated the saturation magnetization dependence of the SSE in the isostructural spinel Ni-Zn ferrites by varying the $\mathrm{Ni}$ and $\mathrm{Zn}$ content.

\section{Experimental procedure}

Samples consist of a series of polycrystalline Ni-Zn ferrites (NZFO) slabs with a mechanically polished surface on which $8.5 \pm 0.5 \mathrm{~nm}$ Pt film was sput- 
tered for ISHE detection. The well polished surface allows flat deposition free from defects at the interface, even thorough different grains as shown in fig. 1(a). All samples were prepared using the standard solid state reaction method, mixing thoroughly the oxides with the proper stoichiometric amounts according to equation:

$$
(1-\mathrm{x}) \mathrm{NiO}+\mathrm{xZnO}+\mathrm{Fe}_{2} \mathrm{O}_{3} \rightarrow \mathrm{Ni}_{1-\mathrm{x}} \mathrm{Zn}_{\mathrm{x}} \mathrm{Fe}_{2} \mathrm{O}_{4}
$$

with $x=0,0.3,0.4,0.5,0.7$ and 1 . The corresponding notation in what follows will be NFO, NZFO_30, NZFO_40, NZFO_50, NZFO_70 and ZFO, where $\mathrm{NFO}$ and ZFO correspond to $\mathrm{Ni}$ and $\mathrm{Zn}$ ferrite respectively, and the number indicates the $\mathrm{Zn}$ percentage of each sample relative to $\mathrm{Ni}$ content. The raw materials were powders of $\mathrm{ZnO}$ (Merk 99\%), $\mathrm{NiO}(99.9 \%)$ and $\alpha-\mathrm{Fe}_{2} \mathrm{O}_{3}$ (Merk $99.9 \%$ ). Resulting mixture were calcined in air at $1150{ }^{\circ} \mathrm{C}$ for $12 \mathrm{~h}$, then were 40 pressed uniaxially into rectangular shaped slabs with dimensions of $7.0 \times 2.0 \times 0.5$ $\mathrm{mm}^{3}$ as shown in fig. 1(b), and finally were sintered at $1300{ }^{\circ} \mathrm{C}$ for $24 \mathrm{~h}$. We performed structural and magnetic characterization by using X-ray diffraction (XRD), Scanning/Transmission electron microscope with high Angular Annular dark field (STEM-HAADF) detector, vibrating sample magnetometry (VSM) and Mössbauer spectroscopy methods.

We performed the SSE measurements in the longitudinal configuration where a spin current is induced from the NZFO slab to the Pt layer due to a temperature difference $(\Delta T)$ applied across the NZFO/Pt bilayer structure (z-direction) in the presence of a magnetic field parallel to the interface (x-direction). The 50 spin current is converted into an electric voltage (y-direction) by means of the ISHE in the Pt layer. Nowadays it is known that effects of temperature difference in the thermal contacts can affect the magnitude of the SSE signal, as was recently demonstrated by Sola et al.[32]. We also measured the anomalous Nernst effect (ANE)[33] in the FM, and the proximity anomalous Nernst effect

55 (PANE) in the deposited NM in order to rule out spurious signals. ANE was the same geometric configuration as the SSE. The absence of the proximity induced anomalous Nernst effect was also confirmed by measurements under an in-plane 
thermal gradient and a magnetic field applied perpendicular to the NZFO/Pt interface[34]. More details are available in the previous study mentioned above

60 in Ref. [7].

\section{Results and Discussion}

The XRD patterns indicate the formation of a single phase of pure spinel structures [fig. 1(c)] with the configuration $\left[\mathrm{Zn}_{\mathrm{x}} \mathrm{Fe}_{1-\mathrm{x}}\right]_{\mathrm{A}}\left[\mathrm{Ni}_{1-\mathrm{x}} \mathrm{Fe}_{1+\mathrm{x}}\right]_{\mathrm{B}} \mathrm{O}_{4}$, where $\mathrm{A}$ and $\mathrm{B}$ denote tetrahedral and octahedral sites respectively, in the $\mathrm{AB}_{2} \mathrm{O}_{4}$

65 spinel structure[35]. The parameter $\delta=1-\mathrm{x}$ determined the degree of inversion of the structure, thus for $\delta=0(\mathrm{x}=1)$ we have normal spinel structure (ZFO); for $\delta=1(\mathrm{x}=0)$ inverse spinel (NFO); and for intermediate values $(0 \leq \delta \leq 1)$ mixed spinel (NZFO). The magnetic properties are directly dependent on this parameter $\delta$ as shown below. In particular a small $\delta$ value, less than 0.04 , is 70 responsible for a weak ferromagnetic behavior in our samples of ZFO (see Ref. $[7])$.

The diffraction peaks shift towards lower $2 \theta$ values as a function of $\mathrm{Zn}$ content, indicating an increase of lattice parameter. The refined values of lattice parameter [fig. 1(d)] display a linear dependence with Zn concentration. We can

75 explain this behavior by adding the different ionic radii $[36]\left(\left[\mathrm{Zn}^{2+}\right]_{A}=0.600 \AA\right.$; $\left[\mathrm{Fe}^{3+}\right]_{A}=0.490 \AA ;\left[\mathrm{Ni}^{2+}\right]_{B}=0.690 \AA ;\left[\mathrm{Fe}^{3+}\right]_{B}=0.645 \AA$ ) for a unit formula of NZFO as follows:

$\mathrm{x}\left[\mathrm{Zn}^{2+}\right]_{\mathrm{A}}+(1-\mathrm{x})\left[\mathrm{Fe}^{3+}\right]_{\mathrm{A}}+(1-\mathrm{x})\left[\mathrm{Ni}^{2+}\right]_{\mathrm{B}}+(1+\mathrm{x})\left[\mathrm{Fe}^{3+}\right]_{\mathrm{B}}=1.825+0.065 \mathrm{x}$,

which indicates that the total ionic radius linearly increases when $\mathrm{Zn}$ is incorposo rated into the structure. The same dependence has been previously reported[37, $38,39,40]$, but with a slightly different interpretation.

We study the crystal structure of all the samples by mean of STEM-HAADF. fig. 2(a) shows a general profile of lamella $\mathrm{Ni}_{0.6} \mathrm{Zn}_{0.4} \mathrm{Fe}_{2} \mathrm{O}_{4}$. Coherent grains 
(a)

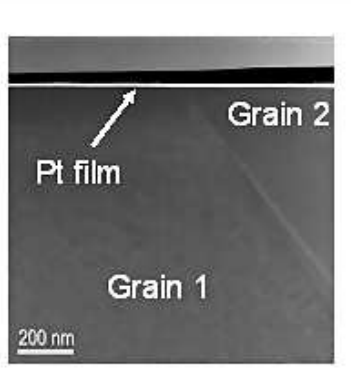

(b)
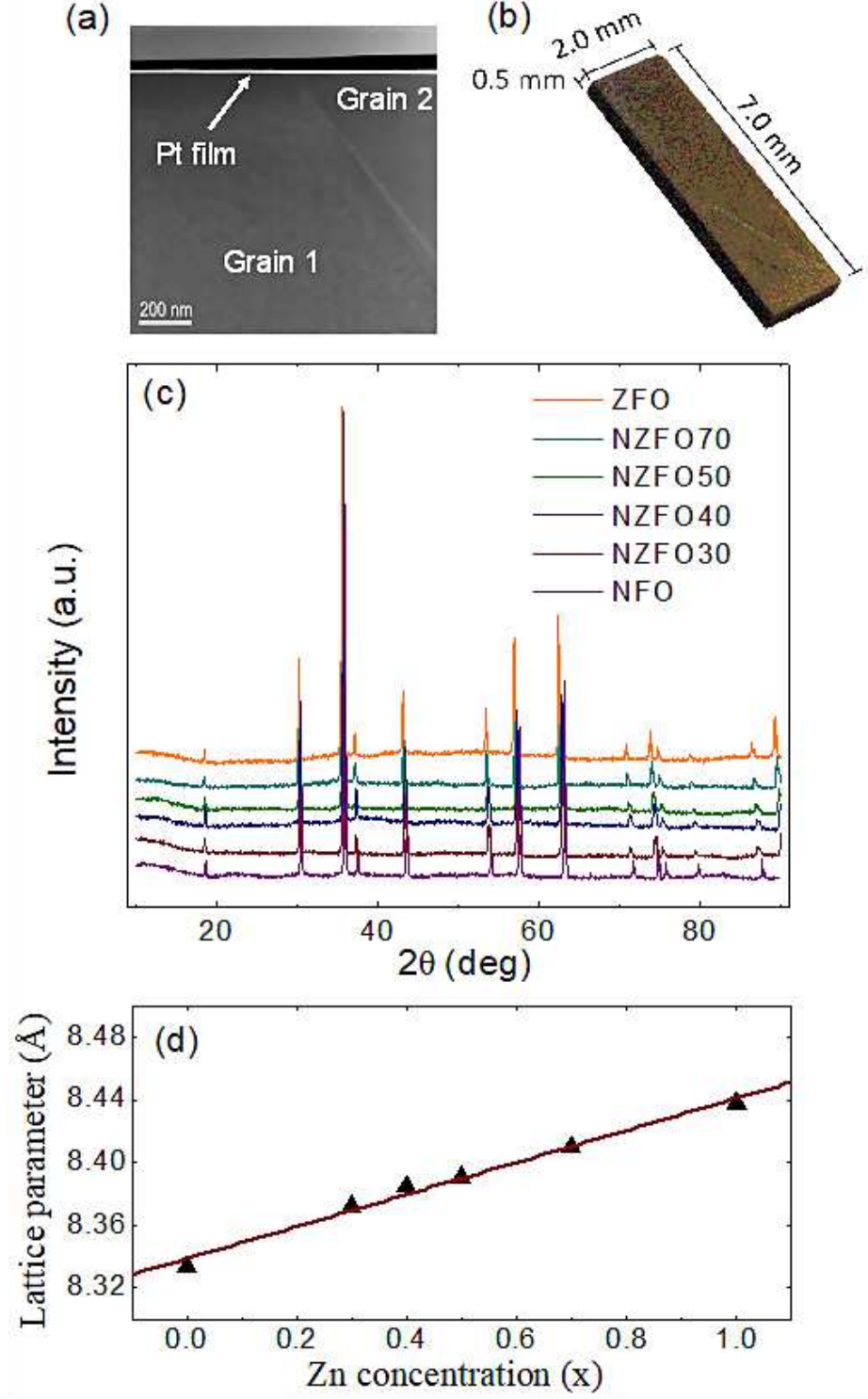

Figure 1: (Color online) (a) Detail of Platinum depostion. Thickness of Pt layer $\approx 8.5 \mathrm{~nm}$. (b) Photograph of a uniaxially pressed and sintered sample after polishing. (c) XRD patterns of $\mathrm{Ni}-\mathrm{Zn}$ ferrites. Zn content increases from bottom to top. (d) Zn concentration dependence of lattice parameter. 


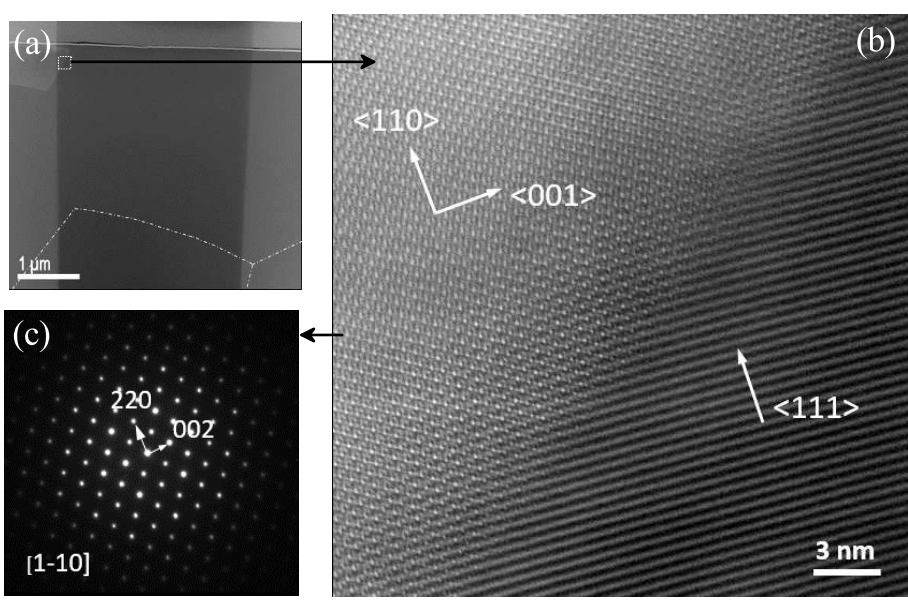

Figure 2: (Color online) (a) General view profile of lamella $\mathrm{Ni}_{0.6} \mathrm{Zn}_{0.4} \mathrm{Fe}_{2} \mathrm{O}_{4}$ with Pt deposition and defined grain boundaries (dotted line). (b) ) STEM-HAADF high resolution image showing the grain boundary detail (c) diffraction pattern of the left grain in b).

with a size of several microns can be clearly distinguished. The Pt layer is composed by nanograins in the range of 5-10 nm [see fig. 1(a)]. High resolution STEM-HAADF image of a grain boundary [fig. 2(b)] shows two typical grain orientations in perfect agreement with spinel structure, as confirmed by the diffraction pattern in fig. 2(c).

Atomic percentages obtained by SEM-EDX are in accordance with the nom90 inal composition as shown in Table 1. An EDS analysis using TEM was performed on a $1 \mu \mathrm{m}$ line through a grain boundary in all samples. The elemental composition is homogeneous despite passing through two different grains. It can be ensured that there are no significant structural defects in grain boundaries.

The magnetization results [fig. 3(a)] show that all NZFO slabs present a 95 soft magnetic behavior with low coercive fields for all compositions. A nonmonotonic variation of saturation magnetization depending on the inversion parameter $\delta$ is presented in fig. 3(b). Magnetic measurements were made before and after Pt deposition. As can be seen in fig. 3(c), the NZFO slabs show a magnetic anisotropy with in plane easy axis. Aditionally, we ensure that Pt 
100 film deposited on the top surface does not significantly affect the bulk magnetic properties of the sample. Magnetism in spinel ferrites is well explained on reference [35]. In a complete normal spinel structure $(\delta=1)$, ZFO presents paramagnetic behavior at room temperature and have a Neel transition around $9 \mathrm{~K}$. However for a small grade of inversion in the spinel structure $(\delta \leq 4 \%) \mathrm{ZFO}$ exhibit weak ferromagnetism[7]. We measure magnetization saturation of about $1.2 \mathrm{emu} / \mathrm{g}$ [inset fig. 3(a)] in the ZFO sample. Therefore, by variation of $\mathrm{Zn}$ content, NZFO mixed spinel ferrites allow magnetization tuning in isostructural samples.

Room-temperature Mössbauer spectra of the investigated samples are displayed in fig. 4. In NFO sample, B sites are occupied mainly by $\mathrm{Ni}^{2+}$ ions and thus half of the $\mathrm{Fe}^{3+}$ ions occupy A sites while the other half $\mathrm{B}$ sites, forming an inverse spinel. The spectrum consist of two well defined sextets with isomer shifts (IS) of $0.25 \mathrm{~mm} / \mathrm{s}$ and $0.36 \mathrm{~mm} / \mathrm{s}$, which are consistent with the presence of Fe (III) in two different sites[41]. For ZFO sample, the preference of $\mathrm{Zn}^{2+}$ ions to occupy A sites requires all $\mathrm{Fe}^{3+}$ ions occupy $\mathrm{B}$ sites in a normal spinel structure. Therefore, the $\mathrm{Fe}^{3+}$ ions in the A sublattice is surrounded by non-magnetic ions in the B sublattices, resulting in a doublet with IS of 0.36 $\mathrm{mm} / \mathrm{s}$ and quadrupole splitting (QS) of $0.32 \mathrm{~mm} / \mathrm{s}$ in agreement with previous reports[42]. The absence of at least one magnetic sextet indicates that the

Table 1: SEM measured of atomic percentages in NZFO slabs

\begin{tabular}{lcccc} 
Sample & \multicolumn{5}{c}{ at $\%$} \\
& $\mathrm{Ni}$ & $\mathrm{Zn}$ & $\mathrm{Fe}$ & $\mathrm{O}$ \\
\hline NFO & 14.81 & - & 28.72 & 56.47 \\
NZFO_30 & 10.52 & 2.89 & 29.44 & 57.16 \\
NZFO_40 & 8.82 & 5.09 & 29.51 & 56.57 \\
NZFO_50 & 7.30 & 6.40 & 30.28 & 56.02 \\
NZFO_70 & 4.64 & 9.27 & 30.70 & 56.38 \\
ZFO & - & 13.06 & 30.27 & 56.66
\end{tabular}



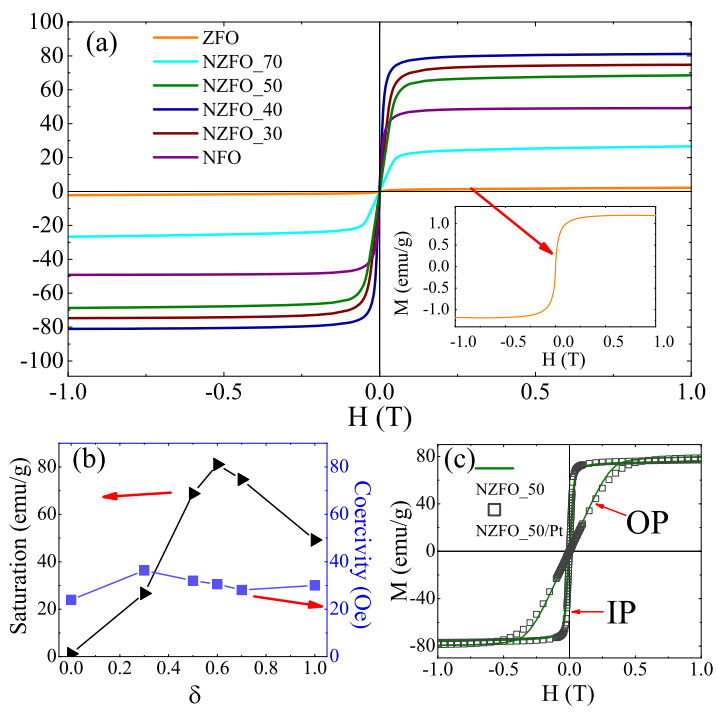

Figure 3: (Color online) (a) Magnetic field dependence of magnetization in NZFO samples. (inset) enlargement of ZFO M-H curve. (b) Saturation magnetization (triangles) and coercive fields (squares) as a function of the inversion parameter $\delta$. (c) In plane (IP) and out of plane (OP) magnetic measurements in NZFO_50, before (continuous line) and after Pt deposition (empty squares symbol).

inversion parameter is very small $(\delta<0.04)$, below the Mössbauer detection limit. In the mixed spinel NZFO ferrites, the best spectra fitting were obtained introducing multiple sextets in the B sites, by using a random distribution based model[43] of $\mathrm{Zn}^{2+}$ and $\mathrm{Fe}^{3+}$ in A sites. Spectra consist of a well defined sextet in A sites (Sext1-A) where all the neighbors of $\mathrm{Fe}^{3+}$ are magnetic $\left(\mathrm{Fe}^{3+}\right.$ or ${ }_{125} \mathrm{Ni}^{2+}$ ); and various sextets in $\mathrm{B}$ sites, with different values of the hyperfine magnetic field $\left(\mathrm{H}_{\text {eff }}\right)$, due to the different magnetic environments of $\mathrm{Fe}^{3+}$, caused by the random distribution of the magnetic ions in the A sites. In NZFO_70 one doublet appears indicating that some $\mathrm{Fe}^{3+}$ ions in $\mathrm{B}$ sites have no magnetic neighbors as a result of the increased Zn content. We list the Mössbauer 130 hyperfine parameters in Table 2. We calculate $\delta$ from spectra fit parameters, obtaining the expected values according to the samples stoichiometry. 

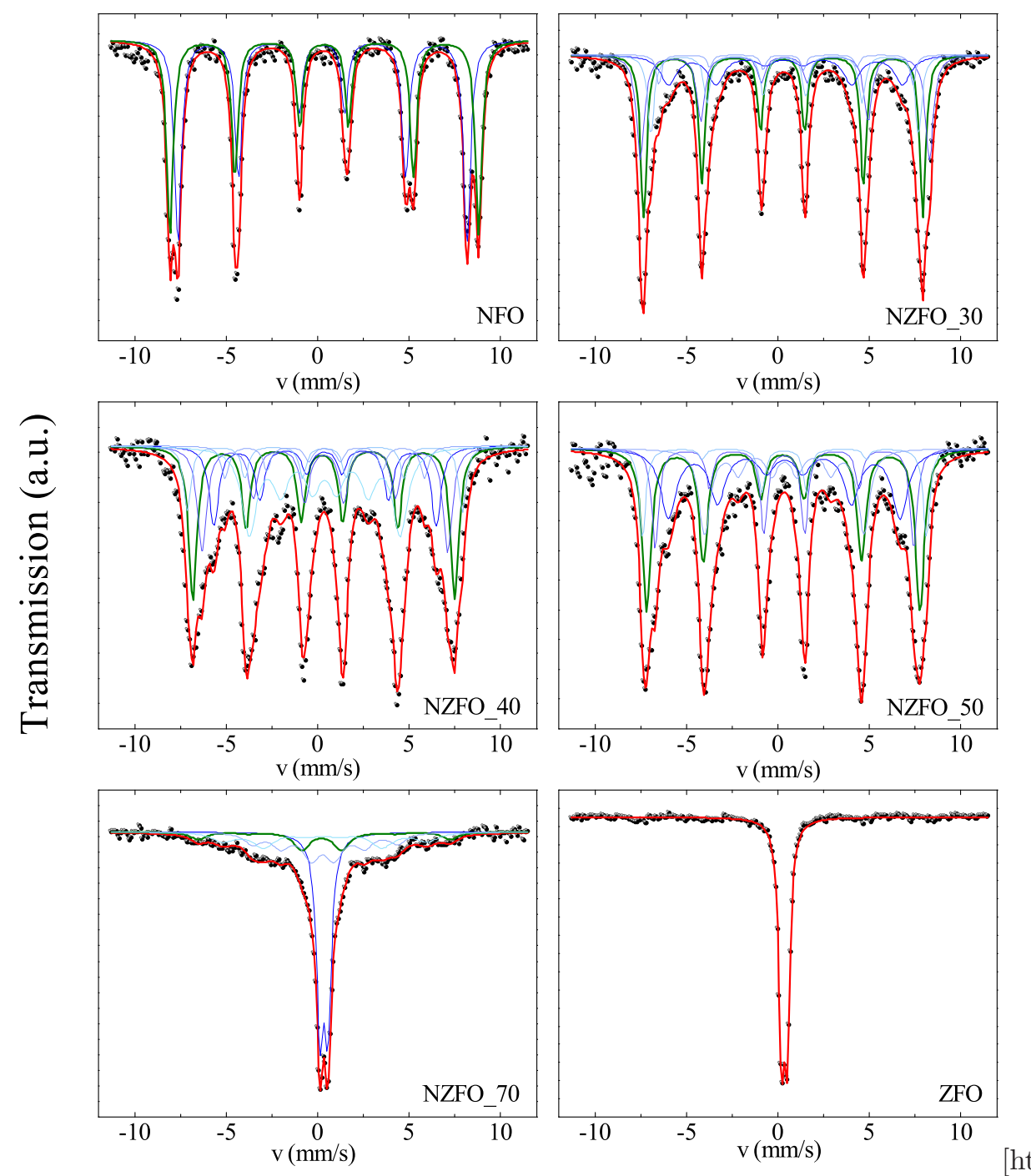

Figure 4: (Color online) Mössbauer spectra of Ni-Zn ferrites. Black points are the experimental data. Red line is the fit. Green line represent the well defined state of $\left[\mathrm{Fe}^{3+}\right]_{\mathrm{A}}$ ions. The different blue lines represent various magnetic environments of $\left[\mathrm{Fe}^{3+}\right]_{\mathrm{B}}$ ions. 
fig. 5(a) shows the schematic illustration of the measurement setup for the SSE. The SSE transversal voltage $V$ as function of the applied magnetic field $H$ at room temperature for the spinel NZFO/Pt samples is shown in fig. 5(b). SSE signal was normalized by sample geometry $\left(L_{z} / L_{y}\right)$ and temperature difference $(\Delta T)$. In what follows we will discuss about the coercive magnetic field present in all samples and the independence of the SSE signal with the saturation magnetization.

(a)

(b)
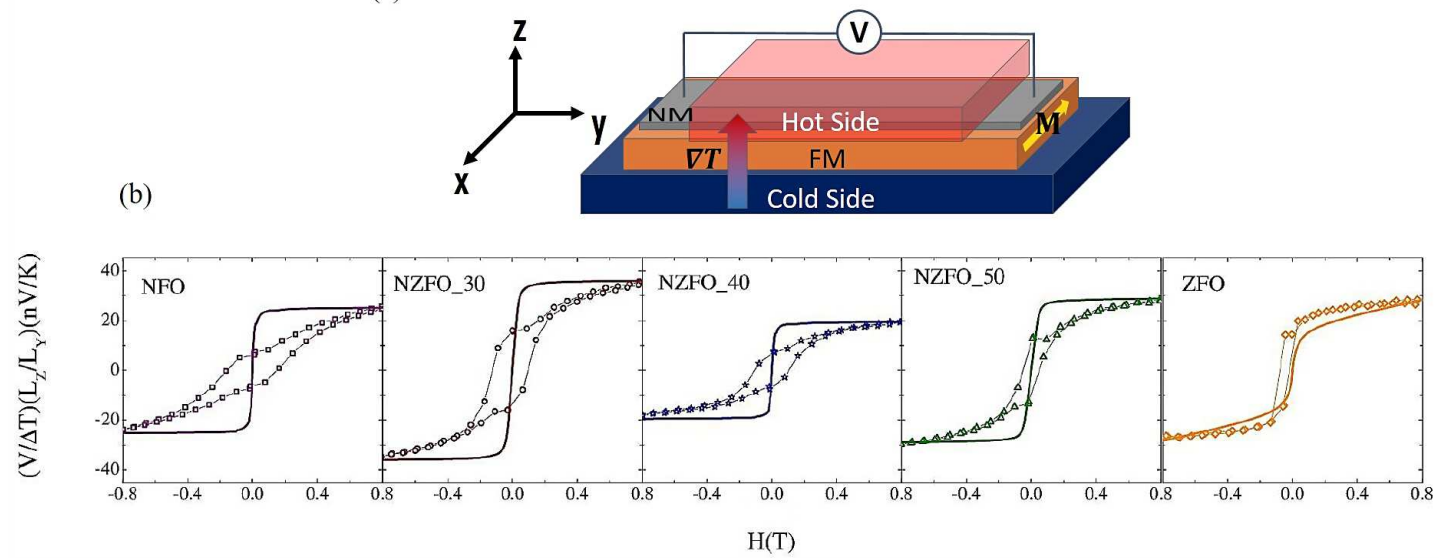

Figure 5: (Color online) (a) Setup to SSE measurement. The temperature gradient is applied to the FM in z-direction, perpendicular to both the FM/NM interface and sample magnetization which is parallel to the applied magnetic field in $\mathrm{x}$-direction. The response signal is measured at the ends of the NM according to the ISHE in y-direction. (b) Magnetic field dependence of the SSE voltage for spinel NZFO samples with different Zn concentration (open figures) and the comparison with its corresponding normalized magnetization curve (solid line). Magnetization curves are properly scaled to the maximum values of SSE voltage in order to visually compared both of them.

\subsection{Magnetic coercive field: The role of surface roughness}

An hysteretic loop is observed in all NZFO/Pt samples with a notable coercivity of up to $0.3 \mathrm{~T}$ approximately which differs significantly from the bulk magnetization dependence. Similar results were found in Mn-Zn ferrite slabs by Uchida et al.[13]. They claimed that voltage signal comes from surface magne- 
tization and suggest the SSE as surface magnetization probe. Saiga et al.[15] also report an hysteretic behavior in a polycrystalline YIG slab. A different magnetic field dependence of the SSE and the magnetization was found in the first measure of SSE by Uchida in a single crystal YIG micrometer slab[5]. In this system the voltage does not describe an hysteresis loop but it is suppressed in the low magnetic field regime. They also demonstrated that the origin of this behavior is due to an intrinsic surface magnetic anisotropy and is not affected by YIG/Pt interface[25]. Aqeel et al.[26] studied the influence of the interface quality on the SSE of the bilayer system single crystal YIG/Pt. They prepared three different types of surfaces and could explained the suppression of the SSE signal at low magnetic fields considering the effect of the perpendicular anisotropy. They also showed that the hysteretic behavior is due to the surface roughness and determined that the coercive field increases with the interface roughness. Therefore, since NZFO were mechanically polished they must have a high degree of roughness and a hysteresis loop appears in the SSE voltage when the magnetic field is swept, according to the above reports.

\subsection{Spin seebeck response independence on saturation magnetization}

The linear dependence of the voltage $V_{\mathrm{SSE}}=(V(0.8 \mathrm{~T})-V(-0.8 \mathrm{~T})) / 2$ as a function of the temperature difference $\Delta T$ is shown in fig. 6(a). We obtain the SSE response from the slope of these lines. We can see in fig. 6(b) that all points are within the range of $25.5 \pm 5.4 \mathrm{nV} / \mathrm{K}$, suggesting that the signal is not dependent on the inversion parameter $\delta$.

A systematic study in various ferrimagnetic garnet ferrites $\mathrm{Y}_{3-\mathrm{x}} \mathrm{R}_{\mathrm{x}} \mathrm{Fe}_{5-\mathrm{y}} \mathrm{M}_{\mathrm{y}} \mathrm{O}_{12}$ $(\mathrm{R}=\mathrm{Gd}, \mathrm{Ca} ; \mathrm{M}=\mathrm{Al}, \mathrm{Mn}, \mathrm{V}, \mathrm{In}, \mathrm{Zr})$ performed by Uchida et al.[14] showed a positive correlation between $M_{s}$ and the SSE response. They claim that $M_{s}$ is directly related to $\mathrm{Fe}$ content in this samples and therefore the spin-mixing conductance monotonically increases with increasing magnetic moment density at the NM/FM interface. Although previous argument comes from the interface, It is unclear what the surface distribution of magnetic moments from Fe is. Otherwise, Shan et al. very recently observed a closely related SSE signal 

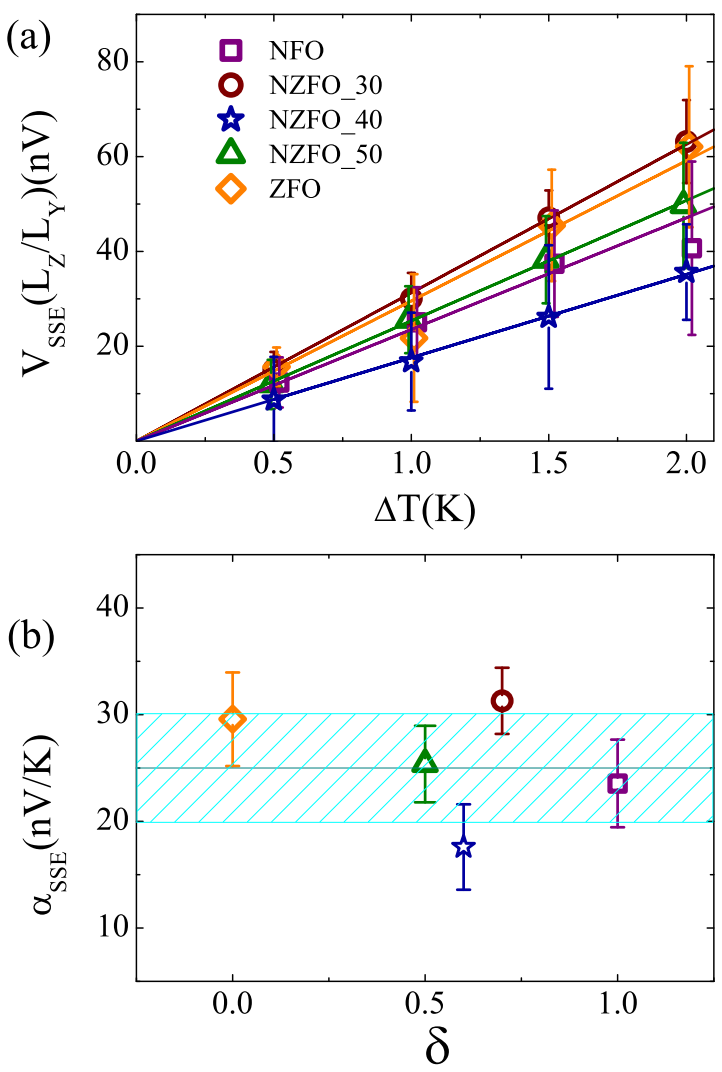

Figure 6: (Color online) (a) Temperature difference dependence of the maximum voltage. (b) Estimated spin Seebeck response as a function of the structural inversion parameter.

with magnetic behaviour in NFO/Pt samples[31].

fig. 6(b) shows the spin Seebeck response $\alpha_{S S C}$ as a function of the inversion parameter $\delta$, exhibiting no sign of correlation between both parameters. As the samples have similar resistivity, we compare the voltage directly. The Fe atomic percentage remains constant in the samples for all $\mathrm{Zn}$ concentrations as can be seen on Table 1. However, saturation magnetization $M_{s}$ in our samples shows no positive correlation with Fe content, instead it depends on $\delta$ which varies magnetization not monotonously (as described above) in the isostructural series of NZFO slabs. It is then assumed that there is no trivial dependence of the $\alpha_{S S C}$ with the macroscopic magnetic parameters such $M_{s}$. Moreover, the 
negligible $M_{s}$ value in ZFO discards any correlation with SSE response voltage. Measurements in thin films would also help to get a insight on this issue. Nevertheless, we observed that the SSE has no bulk magnetization dependence in the isostructural set of NZFO spinel slabs by measurement the SSE voltage in samples with different $\delta$ values, allowing $M_{s}$ variations. However, the SSE signal could be correlated to surface magnetization as in YIG slabs[27]. Moreover, as the spin mixing conductance depends on the magnetic density at the interface[44, 45], it could be important in the SSE voltage response, even in our samples, where the Fe content and the crystalline structure remain unchanged, and the surface of the whole set was polished following the same experimental protocol.

\section{Summary}

We systematically studied the SSE in a series of polycristalline NZFO spinel slabs by means of the ISHE. Samples have a variable $M_{s}$ depending on the structural inversion parameter $\delta$, however the spin Seebeck response remains constant in the entire series of samples.

\section{5. acknowledgments}

The authors acknowledge Professor P. Algarabel, Dr. I. Lucas, and Professor L. Morellón for enlighted discussion. This work was supported by Solid State Group (GES) at the University of Antioquia in the framework of Sustainability Strategy 2016-2017; Colombian Science, technology and innovation department (COLCIENCIAS, PhD student grant, conv. 567); Municipality of Medellín through SAPIENCIA agency (EnlazaMundos program, conv. 2014). We also thank the Spanish Ministry of Science (through and MAT2014-51982-C2-R, including FEDER funding); the Aragón Regional Government (Project No. E26); and Thermo- Spintronic Marie Curie CIG (Grant Agreement No. 304043)- EU. 210 Project No. PRI-PIBJP-2011-0794. This work was also supported by JST ERATO Grant Number JPMJER1402 from JST, Japan. 


\section{References}

[1] K. Uchida, M. Ishida, T. Kikkawa, A. Kirihara, T. Murakami, E. Saitoh, Longitudinal spin seebeck effect: from fundamentals to applications,

J. Phys.: Condens. Matter. $26 \quad$ (34) (2014) 343202. doi : 10.1088/0953-8984/26/34/343202.

URL http://stacks.iop.org/0953-8984/26/i=34/a=343202

[2] G. E. W. Bauer, E. Saitoh, B. J. van Wees, Spin caloritronics, Nat. Mater. 11 (2012) 391. doi:10.1038/nmat3301. URL http://dx.doi.org/10.1038/nmat3301

[3] S. R. Boona, R. C. Myers, J. P. Heremans, Spin caloritronics, Energy Environ. Sci 7 (2014) 885-910. doi:10.1039/C3EE43299H.

URL http://dx.doi.org/10.1039/C3EE43299H

[4] R. Ramos, T. Kikkawa, K. Uchida, H. Adachi, I. Lucas, M. H. Aguirre, P. Algarabel, L. Morellón, S. Maekawa, E. Saitoh, M. R. Ibarra, Observation of the spin seebeck effect in epitaxial fe3o4 thin films, Appl. Phys. Lett. 102 (7) (2013) -. doi:10.1063/1.4793486.

URL http: //scitation.aip.org/content/aip/journal/apl/102/7/10.1063/1.4793486

[5] K. Uchida, H. Adachi, T. Ota, H. Nakayama, S. Maekawa, E. Saitoh, Observation of longitudinal spin-seebeck effect in magnetic insulators, Appl. Phys. Lett. 97 (17). doi:10.1063/1.3507386. URL http://scitation.aip.org/content/aip/journal/apl/97/17/10.1063/1.3507386

[6] K. Uchida, J. Xiao, H. Adachi, J. Ohe, S. Takahashi, J. Ieda, T. Ota, Y. Kajiwara, H. Umezawa, H. Kawai, G. E. W. Bauer, S. Maekawa, E. Saitoh, Spin seebeck insulator, Nat. Mater. 9 (2010) 894. doi:10.1038/NMAT2856. URL http://dx.doi.org/10.1038/nmat2856

[7] J. D. Arboleda, O. A. Olmos, M. H. Aguirre, R. Ramos, A. Anadon, M. R. Ibarra, Spin seebeck effect in a weak ferromagnet, Applied Physics Letters 108 (23) (2016) 232401. arXiv:http://dx.doi.org/10.1063/1.4953229, 
doi $: 10.1063 / 1.4953229$.

URL http://dx.doi.org/10.1063/1.4953229

[8] S. M. Wu, W. Zhang, A. KC, P. Borisov, J. E. Pearson, J. S. Jiang, D. Lederman, A. Hoffmann, A. Bhattacharya, Antiferromagnetic spin seebeck effect, Phys. Rev. Lett. 116 (2016) 097204. doi:10.1103/PhysRevLett.116.097204.

URL http://link.aps.org/doi/10.1103/PhysRevLett.116.097204

[9] S. Seki, T. Ideue, M. Kubota, Y. Kozuka, R. Takagi, M. Nakamura, Y. Kaneko, M. Kawasaki, Y. Tokura, Thermal generation of spin current in an antiferromagnet, Phys. Rev. Lett. 115 (2015) 266601. doi:10.1103/PhysRevLett.115.266601. URL http://link.aps.org/doi/10.1103/PhysRevLett.115.266601

[10] S. M. Wu, J. E. Pearson, A. Bhattacharya, Paramagnetic spin seebeck effect, Phys. Rev. Lett. 114 (2015) 186602. doi:10.1103/PhysRevLett.114.186602.

URL http://link.aps.org/doi/10.1103/PhysRevLett.114.186602

[11] R. Ramos, T. Kikkawa, M. H. Aguirre, I. Lucas, A. Anadón, T. Oyake, K. Uchida, H. Adachi, J. Shiomi, P. A. Algarabel, L. Morellón, S. Maekawa, E. Saitoh, M. R. Ibarra, Unconventional scaling and significant enhancement of the spin seebeck effect in multilayers, Phys. Rev. B 92 (2015) 220407. doi:10.1103/PhysRevB.92.220407.

URL https://link.aps.org/doi/10.1103/PhysRevB.92.220407

[12] R. Ramos, T. Kikkawa, A. Anadn, I. Lucas, K. Uchida, P. A. Algarabel, L. Morelln, M. H. Aguirre, E. Saitoh, M. R. Ibarra, Temperature dependence of the spin seebeck effect in [fe3o4/pt]n multilayers, AIP Advances 7 (5) (2017) 055915. arXiv: http://dx.doi.org/10.1063/1.4974060, doi:10.1063/1.4974060.

URL http://dx.doi.org/10.1063/1.4974060 
[13] K. Uchida, T. Nonaka, T. Ota, E. Saitoh, Longitudinal spin-seebeck effect in sintered polycrystalline (mn,zn)fe2o4, Appl. Phys. Lett. $\quad 97$ doi:http://dx.doi.org/10.1063/1.3533397.

URL http://scitation.aip.org/content/aip/journal/apl/97/26/10.1063/1.3533397

[14] K. Uchida, T. Nonaka, T. Kikkawa, Y. Kajiwara, E. Saitoh, Longitudinal spin seebeck effect in various garnet ferrites, Phys. Rev. B 87 (2013) 104412. doi:10.1103/PhysRevB.87.104412.

URL http://link.aps.org/doi/10.1103/PhysRevB.87.104412

[15] Y. Saiga, K. Mizunuma, Y. Kono, J. C. Ryu, H. Ono, M. Kohda, E. Okuno, Platinum thickness dependence and annealing effect of the spin-seebeck voltage in platinum/yttrium Appl. Phys. Express 7 (9) (2014) 093001. URL http://stacks.iop.org/1882-0786/7/i=9/a=093001

[16] S. R. Boona, K. Vandaele, I. N. Boona, D. W. McComb, J. P. Heremans, Observation of spin seebeck contribution to the transverse thermopower in ni-pt and mnbi-au bulk n Nature Communications 7 (2016) 13714 EP -, article.

URL http://dx.doi.org/10.1038/ncomms13714

[17] A. Hoffmann, Spin hall effects in metals, Magnetics, IEEE Transactions on 49 (10) (2013) 5172-5193. doi:10.1109/TMAG. 2013. 2262947.

[18] J. Xiao, G. E. W. Bauer, K.-c. Uchida, E. Saitoh, S. Maekawa, Theory of magnon-driven spin seebeck effect, Phys. Rev. B 81 (2010) 214418. doi:10.1103/PhysRevB.81.214418. URL https://link.aps.org/doi/10.1103/PhysRevB.81.214418

[19] H. Adachi, J.-i. Ohe, S. Takahashi, S. Maekawa, Linear-response theory of spin seebeck effect in ferromagnetic insulators, Phys. Rev. B 83 (2011) 094410. doi:10.1103/PhysRevB.83.094410. URL http://link.aps.org/doi/10.1103/PhysRevB .83.094410 
[20] S. M. Rezende, R. L. Rodríguez-Suárez, R. O. Cunha, A. R. Rodrigues, F. L. A. Machado, G. A. Fonseca Guerra, J. C. Lopez Ortiz, A. Azevedo, Magnon spin-current theory for the longitudinal spin-seebeck effect, Phys. Rev. B 89 (2014) 014416. doi:10.1103/PhysRevB.89.014416.

URL https://link.aps.org/doi/10.1103/PhysRevB.89.014416

[25] K. Uchida, J.-i. Ohe, T. Kikkawa, S. Daimon, D. Hou, Z. Qiu, E. Saitoh, Intrinsic surface magnetic anisotropy in $\mathrm{y}_{3} \mathrm{fe}_{5} \mathrm{O}_{12}$ as the origin of low-magnetic-field behavior of the 
Phys. Rev. B 92 (2015) 014415. doi:10.1103/PhysRevB.92.014415.

URL http://link.aps.org/doi/10.1103/PhysRevB.92.014415

325

[26] A. Aqeel, I. J. Vera-Marun, B. J. van Wees, T. T. M. Palstra, Surface sensitivity of the spin seebeck effect, Journal of Applied Physics 116 (15). doi:10.1063/1.4897933.

URL http: //scitation. aip.org/content/aip/journal/jap/116/15/10.1063/1.4897933

[27] V. Kalappattil, R. Das, M.-H. Phan, H. Srikanth, Roles of bulk and surface magnetic anisotropy on the longitudinal spin seebeck effect of pt/yig, Scientific Reports 7 (1) (2017) 13316. doi:10.1038/s41598-017-13689-2. URL https://doi.org/10.1038/s41598-017-13689-2

[28] E.-J. Guo, A. Herklotz, A. Kehlberger, J. Cramer, G. Jakob, M. Klui, Thermal generation of spin current in epitaxial cofe2o 4 thin films, Applied Physics Letters $108 \quad$ (2) (2016) 022403. arXiv:https://doi.org/10.1063/1.4939625, doi:10.1063/1.4939625. URL https://doi.org/10.1063/1.4939625

[29] T. Niizeki, T. Kikkawa, K. ichi Uchida, M. Oka, K. Z. Suzuki, H. Yanagihara, E. Kita, E. Saitoh, Observation of longitudinal spin-seebeck effect in cobalt-ferrite epitaxial thin films, AIP Advances 5 (5) (2015) 053603. arXiv: https ://doi.org/10.1063/1.4916978, doi:10.1063/1.4916978.

URL https://doi.org/10.1063/1.4916978

[30] D. Meier, T. Kuschel, L. Shen, A. Gupta, T. Kikkawa, 345 K. Uchida, E. Saitoh, J.-M. Schmalhorst, G. Reiss, Thermally driven spin and charge currents in thin nife $\mathrm{O}_{4} / \mathrm{pt}$ films, Phys. Rev. B 87 (2013) 054421. doi:10.1103/PhysRevB.87.054421. URL http://link.aps.org/doi/10.1103/PhysRevB.87.054421

[31] J. Shan, P. Bougiatioti, L. Liang, G. Reiss, T. Kuschel, B. J. van Wees, Nonlocal magnon spin transport in nife2o4 thin films, Applied 
Physics Letters 110 (13) (2017) 132406. doi:10.1063/1.4979408.

URL https://doi.org/10.1063/1.4979408

[32] A. Sola, P. Bougiatioti, M. Kuepferling, D. Meier, G. Reiss, M. Pasquale, T. Kuschel, V. Basso, Longitudinal spin seebeck coefficient: heat flux vs. temperature difference method, Scientific Reports 7 (2017) 46752 EP -, article.

URL http://dx.doi.org/10.1038/srep46752

[33] T. Miyasato, N. Abe, T. Fujii, A. Asamitsu, S. Onoda, Y. Onose, N. Nagaosa, Y. Tokura,

Crossover behavior of the anomalous hall effect and anomalous nernst effect in itinerant ferromagnet Phys. Rev. Lett. 99 (2007) 086602. doi : 10.1103/PhysRevLett.99.086602.

URL http://link.aps.org/doi/10.1103/PhysRevLett.99.086602

[34] T. Kikkawa, K. Uchida, Y. Shiomi, Z. Qiu, D. Hou, D. Tian, H. Nakayama, X.-F. Jin, E. Saitoh, Longitudinal spin seebeck effect free from the proximity nernst effect, Phys. Rev. Lett. 110 (2013) 067207. doi:10.1103/PhysRevLett.110.067207. URL http://link.aps.org/doi/10.1103/PhysRevLett.110.067207

[35] D. S. Mathew, R.-S. Juang, An overview of the structure and magnetism of spinel ferrite nanoparticle Chem. Eng. J. $129 \quad$ (13) (2007) $51 \quad$ - 65. doi:http://dx.doi.org/10.1016/j.cej.2006.11.001.

URL http://www.sciencedirect.com/science/article/pii/S1385894706004931

[36] R. D. Shannon, Revised effective ionic radii and systematic studies of interatomic distances in halides Acta Crystallographica Section A 32 (5) (1976) 751-767. doi:10.1107/S0567739476001551. URL http://dx.doi.org/10.1107/S0567739476001551

[37] G. Srinivasan, E. T. Rasmussen, R. Hayes, Magnetoelectric effects in ferrite-lead zirconate titanate layered composites: The influence of zinc sul Phys. Rev. B 67 (2003) 014418. doi:10.1103/PhysRevB.67.014418.

URL http://link.aps.org/doi/10.1103/PhysRevB .67.014418 
[38] R. Kambale, N. Adhate,

B. Chougule, Y. Kolekar,

Magnetic and dielectric properties of mixed spinel nizn ferrites synthesized by citratenitrate combust Journal of Alloys and Compounds 491 (12) (2010) 372 - 377. doi:http://dx.doi.org/10.1016/j.jallcom.2009.10.187.

URL http://www.sciencedirect.com/science/article/pii/S0925838809021872

[39] M. Sorescu, L. Diamandescu, R. Peelamedu, R. Roy, P. Yadoji, Structural and magnetic properties of nizn ferrites prepared by microwave sintering, Journal of Magnetism and Magnetic Materials 279 (23) (2004) 195 - 201. doi:http://dx.doi.org/10.1016/j.jmmm.2004.01.079.

URL http://www.sciencedirect.com/science/article/pii/S0304885304001428

[40] M. Jalaly, M. Enayati, P. Kameli, F. Karimzadeh,

Effect of composition on structural and magnetic properties of nanocrystalline ball milled ni1xznxfe2 Physica B: Condensed Matter $405 \quad$ (2) (2010) $507 \quad$ - 512. doi:http://dx.doi.org/10.1016/j.physb.2009.09.044.

URL http://www.sciencedirect.com/science/article/pii/S0921452609010801

H. G.

Drickamer, High-pressure studies on ferrites, Phys. Rev. B 10 (1974) 154-164. doi:10.1103/PhysRevB.10.154.

URL http://link.aps.org/doi/10.1103/PhysRevB.10.154

[42] G. Goya, H. Rechenberg, Ionic disorder and nel temperature in znfe2o4 nanoparticles, Journal of Magnetism and Magnetic Materials 196197 (1999) 191 - 192. doi:http://dx.doi.org/10.1016/S0304-8853(98)00723-9. URL http://www.sciencedirect.com/science/article/pii/S0304885398007239

[43] N. Velinov, E. Manova, T. Tsoncheva, C. Estourns, D. Paneva, K. Tenchev, V. Petkova, K. Koleva, B. Kunev, I. Mitov, Spark plasma sintering synthesis of ni1xznxfe2o4 ferrites: Mssbauer and catalytic study,

Solid State Sciences $14 \quad(8) \quad(2012) \quad 1092 \quad$ - 1099.
doi:http://dx.doi.org/10.1016/j.solidstatesciences.2012.05.023. URL http://www.sciencedirect.com/science/article/pii/S1293255812001860 
[44] H. Yuasa, K. Tamae, N. Onizuka, Spin mixing conductance enhancement by increasing magnetic dens AIP Advances 7 (5) (2017) 055928. arXiv:https://doi.org/10.1063/1.4977496, doi:10.1063/1.4977496.

URL https://doi.org/10.1063/1.4977496

[45] X. Jia, K. Liu, K. Xia, G. E. W. Bauer, Spin transfer torque on magnetic insulators, EPL (Europhysics Letters) 96 (1) (2011) 17005.

URL http://stacks.iop.org/0295-5075/96/i=1/a=17005 
Table 2: Mössbauer hyperfine parameters of the investigated samples: Isomer shift (IS), quadrupole splitting (QS), hyperfine magnetic field $\left(\mathrm{H}_{\mathrm{eff}}\right)$, normalized site population $(\%)$ and inversion parameter $(\delta)$

\begin{tabular}{|c|c|c|c|c|c|c|}
\hline Sample & Comp & $\begin{array}{c}\mathrm{IS} \\
(\mathrm{mm} / \mathrm{s})\end{array}$ & $\begin{array}{c}\mathrm{QS} \\
(\mathrm{mm} / \mathrm{s})\end{array}$ & $\begin{array}{l}\mathrm{H}_{\mathrm{eff}} \\
(\mathrm{T})\end{array}$ & $\%$ & $\delta^{1}$ \\
\hline \multirow[t]{2}{*}{$\mathrm{NFO}$} & Sext1-A & 0.25 & 0.01 & 49.1 & 51.1 & 1.02 \\
\hline & Sext2-B & 0.36 & 0.00 & 52.4 & 48.9 & \\
\hline \multirow[t]{5}{*}{ NZFO_30 } & Sext1-A & 0.27 & 0.02 & 47.4 & 35.5 & 0.71 \\
\hline & Sext2-B & 0.37 & 0.05 & 39.8 & 17.0 & \\
\hline & Sext3-B & 0.37 & 0.01 & 49.0 & 24.9 & \\
\hline & Sext4-B & 0.37 & 0.00 & 45.4 & 17.8 & \\
\hline & Sext5-B & 0.37 & 0.07 & 43.2 & 4.7 & \\
\hline \multirow[t]{5}{*}{ NZFO_40 } & Sext1-A & 0.27 & 0.02 & 46.4 & 31.1 & 0.62 \\
\hline & Sext2-B & 0.35 & 0.01 & 39.3 & 24.3 & \\
\hline & Sext3-B & 0.35 & 0.01 & 43.9 & 17.4 & \\
\hline & Sext4-B & 0.35 & 0.00 & 48.2 & 10.3 & \\
\hline & Sext5-B & 0.35 & 0.00 & 27.1 & 16.9 & \\
\hline \multirow[t]{6}{*}{ NZFO_50 } & Sext1-A & 0.29 & 0.05 & 44.4 & 25.7 & 0.51 \\
\hline & Sext2-B & 0.38 & 0.02 & 37.7 & 15.0 & \\
\hline & Sext3-B & 0.38 & 0.02 & 41.7 & 19.4 & \\
\hline & Sext4-B & 0.38 & 0.01 & 46.7 & 9.4 & \\
\hline & Sext5-B & 0.38 & 0.01 & 33.9 & 5.5 & \\
\hline & Sext6-B & 0.38 & 0.00 & 25.6 & 24.9 & \\
\hline \multirow[t]{4}{*}{ NZFO_70 } & Sext1-A & 0.29 & 0.06 & 42.7 & 13.4 & 0.27 \\
\hline & Doub2-B & 0.34 & 0.44 & - & 40.8 & \\
\hline & Sext3-B & 0.34 & 0.07 & 24.6 & 26.6 & \\
\hline & Sext4-B & 0.34 & 0.06 & 34.7 & 19.2 & \\
\hline $\mathrm{ZFO}$ & Doub1-B & 0.36 & 0.32 & - & 100 & $0.0^{2}$ \\
\hline
\end{tabular}

